\title{
Estratégias tecnológicas e desempenhos inovadores das PMEs de equipamentos médico- hospitalares de São Carlos e Ribeirão Preto
}

\author{
Denise Luciana Rieg \\ Centro Universitário Fundação Santo André \\ Alceu Gomes Alves Filho \\ Universidade Federal de São Carlos
}

\begin{abstract}
Resumo
Este trabalho tem como objetivo identificar e analisar as estratégias tecnológicas (ETs) adotadas por pequenas e médias empresas (PMEs) produtoras de equipamentos médico-hospitalares localizadas em São Carlos e em Ribeirão Preto. Procura-se verificar se há diferenças entre o grupo de empresas que alcançou desempenhos inovadores (Dls) mais altos e o grupo daquelas que tiveram Dls mais baixos nos últimos anos. Para isso, foi realizado um survey, aplicando-se um questionário estruturado em trinta e nove empresas que concordaram em participar da pesquisa. Os resultados (resultados referentes a vinte e sete empresas que puderam ser classificadas como sendo de maior ou menor DI, dentre as trinta e nove empresas investigadas] mostram que as ETs adotadas pelas empresas com Dls mais altos são diferentes em muitos aspectos daquelas adotadas pelo grupo com Dls mais baixos. Foram obtidos indícios de que maiores investimentos em atividades internas de P\&D\&E e em fontes externas de tecnologia, maior dedicação à previsão tecnológica preliminar voltada para monitoramento do perfil de produtos e processos e o desenvolvimento de um amplo portfolio de tecnologias de produto e de processo permitem às empresas alcançarem Dls mais significativos.
\end{abstract}

Palavras-chave

Desempenho inovador, estratégia tecnológica, pequenas e médias empresas, equipamentos médico-hospitalares.

\section{Technology strategies and innovation performances of the small and medium-sized firms in the medical equipment industry of São Carlos and Ribeirão Preto}

\begin{abstract}
The goal of this article is to identify and to analyze the technology strategies (TSs] adopted by small and medium-sized firms of the medical equipment sector located in São Carlos and Ribeirão Preto. It seeks to verify whether there are differences between the group of firms that reached higher innovation performances (IPS) and the one with lower (IPS) in recent years. In order to analyze that, data about TSs and IPs were collected from twenty seven medical equipment producers. The statistical analysis of the data shows that the TSs adopted by the medical equipment firms that reached higher IPs are different in many aspects from the ones adopted by those that reached lower IPs. Some indications were obtained that larger investments in internal activities of R\&D\&E development and in external sources of technology, larger dedication to technology forecasting, and development of a wide portfolio of product and process technologies have allowed the firms to reach higher IPs.
\end{abstract}

Key words

Innovation performance, technology strategy, small and medium-sized firms, medical equipment sector. 


\section{INTRODUC̣̃̃O}

Segundo Miller (1988), pouco esforço tem sido despendido para a integração de pesquisas que buscam analisar a relação entre tecnologia e estratégia e entre tecnologia e desempenho. Sabe-se que estratégia e desempenho estão fortemente interligados à tecnologia, porém pouco se sabe sobre como, com algum detalhamento, estratégia, tecnologia e desempenho se influenciam.

Wilbon (1999) resgata também tal observação ao expor que é possível encontrar na literatura apenas poucos estudos empíricos sobre a influência da estratégia tecnológica (ET) no desempenho das empresas. Alguns desses estudos são: Dowling e Mcgee (1994), Zahra (1996), Deeds et al. (1997) e Lanctot e Swan (2000). Como salienta Wilbon (1999), essa escassez de trabalhos aponta para a necessidade de se realizarem novos estudos empíricos na tentativa de se obter melhor compreensão da dinâmica da relação entre ET e desempenho da empresa.

Neste contexto é que o presente artigo procura apresentar uma caracterização das ETs adotadas e dos desempenhos inovadores (DIs) obtidos pelas empresas produtoras de equipamentos médico-hospitalares localizadas nos municípios de São Carlos e Ribeirão Preto nos últimos anos. Mais especificamente, procura-se verificar se aquelas empresas que obtiveram, nos últimos anos, DIs mais expressivos seguiram estratégias tecnológicas diferentes daquelas que obtiveram DIs em níveis mais modestos.

Não se pretende avaliar aqui se as ETs adotadas são determinantes únicas dos DIs obtidos pelas empresas e explicar de que forma se dá a influência da ET sobre o desempenho. Pretende-se apenas avaliar a hipótese de que as empresas, para sustentarem níveis mais altos de DI, fazem determinadas escolhas estratégicas em relação à aquisição, ao desenvolvimento e ao uso de capacidades tecnológicas que se diferenciam daquelas adotadas pelas empresas que não obtiveram, no mesmo período de análise, os mesmos patamares de DI.

Cabe ressaltar que o interesse pelas empresas investigadas deve-se ao nível de especialização do setor médicohospitalar nas regiões de São Carlos e Ribeirão Preto, como apontado pela pesquisa realizada por Fernandes \& Côrtes (1998), que apresenta um mapeamento das empresas de base tecnológica do Estado de São Paulo. Além disso, trata-se de um setor que apresenta elevado dinamismo tecnológico, o que requer, das empresas que pretendem sobreviver neste ambiente, o desenvolvimento de capacidade para introduzirem freqüentemente produtos novos ou melhorados tecnologicamente.

Além de contribuir com a discussão de temas relacionados à ET, busca-se com esta pesquisa alcançar uma compreensão mais acurada de como atuam as empresas do setor médico- hospitalar de São Carlos e Ribeirão Preto, em termos de seus comportamentos inovativos. Como afirmam Furtado \& Souza (2000), há poucos trabalhos acadêmicos sobre esse setor no Brasil e é ainda menor o número de trabalhos que reúnem informações sobre o processo de inovação e capacitação das empresas produtoras de equipamentos médico-hospitalares. Entre os trabalhos analisados no presente estudo sobre o setor médico-hospitalar, encontram-se Furtado (1999), Albuquerque e Cassiolato (2000), Furtado e Souza (2000), Guia de Fornecedores Hospitalares (2001), Gadelha (2002), Oliveira (2002), Telles (2002) e NEPP (2004).

Para o relato dos resultados da pesquisa, são apresentados os conceitos de ET e de DI adotados neste trabalho e as principais expectativas que se tinha sobre as estratégias tecnológicas adotadas pelos grupos das empresas investigadas com níveis mais altos e mais baixos de DI. Depois, discorre-se sobre o método aplicado para o desenvolvimento da pesquisa e, em seguida, são apresentados os resultados obtidos quanto às ETs e aos DIs das empresas do setor de equipamentos médico-hospitalares de São Carlos e Ribeirão Preto. Finalizando o artigo, faz-se uma síntese dos resultados obtidos.

Esta pesquisa refere-se a um projeto de doutorado financiado pela Fapesp (Fundação de Amparo à Pesquisa do Estado de São Paulo).

\section{ESTRATÉGIA TECNOLÓGICA E DESEMPENHO INOVADOR}

O desempenho inovador (DI) da empresa pode ser avaliado pelo número de produtos e processos tecnologicamente modificados, resultantes dos esforços tecnológicos despendidos (QUADROS et al., 1999), e pela parcela de faturamento proveniente da comercialização desses produtos tecnologicamente modificados (SBRAGIA et al., 1998; SOBEET, 2000). O DI é uma importante dimensão do desempenho das empresas, principalmente para aquelas que se encontram em ambientes tecnologicamente dinâmicos, como é o caso do setor médico-hospitalar, em que os ciclos de vida dos produtos são extremamente curtos.

Nesses ambientes mais dinâmicos, as empresas ampliam os seus gastos com P\&D (pesquisa e desenvolvimento), para desenvolver internamente novos produtos e processos; compram ou licenciam tecnologias desenvolvidas por terceiros para incorporá-las em seus produtos e processos; ou, ainda, realizam alianças estratégicas ou de cooperação com outras empresas para conseguir acesso a tecnologias superiores (best practices). Procedem desta maneira visando ampliar os recursos necessários à produção de produtos e processos novos ou melhorados tecnologicamente que potencialmente contribuam para manter segmentos de mercado ou para entrar em novos segmentos de mercado. 
Pode-se, então, definir o DI das empresas como o resultado mais diretos de suas estratégias tecnológicas (ETs), aqui entendidas como as decisões que as empresas tomam em relação à aquisição, ao desenvolvimento e uso de recursos e capacidades tecnológicas (ZAHRA,1996).

Adotando-se tal conceito de ET, são especificamente analisadas aqui as decisões que as empresas investigadas tomaram, ao longo de três anos (2001, 2002 e 2003), em relação ao uso de fontes externas de tecnologia, às atividades internas de $\mathrm{P} \& \mathrm{D} \& \mathrm{E}$ (pesquisa, desenvolvimento e engenharia), à realização de previsão tecnológica preliminar voltada para monitoramento do perfil de produtos e processos, ao desenvolvimento do portifólio de tecnologias e aos investimentos realizados no sistema produtivo para torná-lo mais eficiente, em termos de rapidez e custos operacionais.

Com efeito, com base em resultados encontrados principalmente em estudos empíricos sobre a influência da ET no desempenho das empresas (LANCTOT; SWAN, 2000; WILBON, 1999; ZAHRA, 1996; DOWLING; MCGEE, 1994; SCHERER; HUH, 1992; ALVES, F., 1991; etc.), pode-se formular algumas expectativas em relação às ETs adotadas pelas empresas aqui investigadas, como exposto a seguir.

Primeiramente, em relação às fontes internas de tecnologia, diversos estudos empíricos concluem que os gastos com P\&D influenciam positivamente o desempenho das empresas. Isto se dá, principalmente, por meio da adaptação e criação de tecnologias através de atividades de P\&D e da implementação dessas novas tecnologias nos produtos por elas produzidos e comercializados. Ou seja, os lançamentos de produtos tecnologicamente novos ou melhorados, resultantes dos esforços de pesquisa e desenvolvimento despendidos pelas empresas, têm auxiliado às mesmas a capturar e manter parcelas de mercado e a aumentar sua lucratividade (DOWLING; MCGEE, 1994; DOWLING; RUEFLI, 1992; PEGELS; THIRUMURTHY, 1996; ZAHRA, 1996). Outros estudos têm mostrado que quanto mais dinâmico for o ambiente no qual opera a empresa, maior será a influência das atividades de pesquisa e desenvolvimento no seu desempenho, dada a necessidade de inovar constantemente (SCHERER; HUH, 1992; SPITAL; BICKFORD, 1992).

Considerando a análise de empresas que atuam em ambientes dinâmicos, a expectativa é que os investimentos em atividades de pesquisa e desenvolvimento e também de engenharia (P\&D\&E) realizados por empresas com DIs mais elevados superem aqueles realizados pelas empresas que obtêm DIs mais baixos. A capacidade de repor freqüentemente produtos novos e versões melhoradas dos produtos existentes torna-se muito importante para a sobrevivência das empresas. Mesmo no caso de inovações incrementais, dadas as dificuldades das empresas situadas em economias periféricas em ter acesso a "tecnologias superiores" (FERNANDES et al., 1999), os investimentos em P\&D\&E tornam-se quase que obrigatórios.

Há que se ressaltar que as atividades de P\&D\&E analisadas aqui incluem aquelas levadas a cabo tanto para desenvolver inovações significativas ou incrementais em produtos e processos desenvolvidos totalmente e unicamente dentro da empresa, quanto para dar suporte à aquisição de tecnologias desenvolvidas por terceiros que vão ser incorporadas aos produtos e processos da empresa. É através das atividades de $P \& D \& E$ que as tecnologias adquiridas são adaptadas às condições de conhecimento da empresa e são realizadas inovações incrementais nessas tecnologias, consolidandose, desta forma, a capacidade tecnológica da empresa, que se torna, assim, única à empresa e, portanto, diferente do conhecimento técnico-científico de domínio público (HASENCLEVER; CASSIOLATO, 1998).

\section{入 lém de contribuir com a discussão de temas relacionados à ET, busca-se com esta pesquisa alcançar uma compreensão mais acurada de como atuam as empresas do setor médico-hospitalar de São Carlos e Ribeirão Preto, em termos de seus comportamentos inovativos.}


vimento de inovações incrementais torna-se condição para que as empresas sigam o padrão de suprir as necessidades desses mercados. Entretanto, a literatura indica que o porte da empresa tem de ser relativamente grande, de modo a viabilizar o investimento em inovação e, eventualmente, a obtenção de economias de escala nas atividades de pesquisa e desenvolvimento (ALVES FILHO, 1991). E, além disso, os resultados das atividades internas de pesquisa e desenvolvimento são, em geral, incertos (ZAHRA, 1996). De porte micro ou pequeno, as empresas aqui analisadas devem ponderar se os gastos com atividades internas de P\&D\&E se justificam e, considerando as restrições e os riscos, verificar se fontes externas de tecnologia constituem uma alternativa melhor de investimento. As fontes externas de tecnologia englobam licenciamentos, alianças estratégicas, compra de tecnologias e contratação de outras empresas, universidades e centros de pesquisa para desenvolver tecnologias de produto e processo. como já exposto anteriormente, para as micro e pequenas empresas, talvez a realização de atividades de pesquisa e desenvolvimento e, também, de engenharia em departamentos estruturados (nas próprias empresas) não se justifique, pois essas atividades requerem, em geral, orçamentos vultosos e, além disso, seus resultados são incertos (ZAHRA, 1996). Frente a isso, uma possibilidade já apresentada aqui é a aquisição de tecnologias desenvolvidas por terceiros. Outra ainda é a realização de atividades esporádicas de P\&D\&E. As empresas realizariam atividades esporádicas de P\&D\&E apenas para tentar, eventualmente, suprir alguma necessidade tecnológica do mercado ou fazer modificações incrementais em tecnologias adquiridas a partir de fontes externas à empresa.

O portfolio de tecnologias da empresa é o conjunto de tecnologias de produto e processo em que a empresa tem investido ao longo do tempo. Isto inclui tanto as tecnologias básicas quanto as tecnologias chaves (HARRIS et al. 1996), geradas pela empresa ou que ela tenha adquirido a partir de fontes externas. Como exposto por Burgelman e Rosenbloom (1989) apud Wilbon (1999), as empresas que possuem amplos portfolios de tecnologias estão mais preparadas para desenvolver novos produtos e melhorias em produtos já existentes e, assim, responder às oportunidades e ameaças no mercado.

Neste sentido, espera-se que aquelas empresas que obtiveram maiores DIs detenham a maioria das tecnologias básicas de produto e de processo (tecnologias necessárias a qualquer empresa para competir na indústria específica) e um número considerável de tecnologias chaves (tecnologias de alto valor e únicas à empresa e que, deste

Assim, parte-se nesta pesquisa da expectativa de que a maioria das empresas investigadas utilize preferencialmente fontes externas de tecnologia - conseqüentemente, invista mais em aquisição de tecnologias a partir de fontes externas do que em atividades internas de $\mathrm{P} \& \mathrm{D} \& \mathrm{E}$ - para a incorporação das tecnologias adquiridas em seus produtos e processos. Nesta mesma linha de argumentação, pode-se esperar que aquelas que atingiram DIs mais elevados invistam mais na aquisição de tecnologias a partir de fontes externas em comparação com aquelas que obtiveram, no mesmo período de análise, DIs mais baixos.

Voltando à análise das atividades internas de P\&D\&E, também devido ao porte das empresas (micro e pequenas empresas), há a expectativa de que as empresas, quando realizam tais atividades, o façam de forma esporádica. Como ressaltado por Kleinknecht et al. (1991), a forma mais observada de geração de tecnologias nos casos de empresas de pequeno porte são justamente as atividades informais ou descontínuas de pesquisa e desenvolvimento. Isto porque, modo, sustentam sua vantagem competitiva). Por outro lado, espera-se que as empresas que obtiveram DIs mais baixos não detenham a maioria das tecnologias básicas de produto e de processo e um conjunto expressivo de tecnologias chaves.

A previsão tecnológica preliminar voltada para monitoramento do perfil de produtos e processos refere-se ao monitoramento que a empresa faz do desenvolvimento tecnológico de produtos e processos em seu setor de atuação e em setores correlatos. Desta forma, essa previsão tecnológica preliminar pode ser medida pela freqüência com que a empresa procura identificar e analisar o que ela precisa em termos de tecnologia de produto e de processo para fortalecer seu poder de concorrência e pela freqüência e magnitude com que a empresa procura ficar a par das mudanças tecnológicas na indústria em que atua e nas indústrias correlatas.

De acordo com Zahra (1996), a previsão tecnológica preliminar ajuda a empresa a identificar as mudanças, as ameaças e as oportunidades no ambiente em que atua, as ini- 
ciativas tecnológicas dos seus concorrentes e as tecnologias substitutivas em potencial. Por sua vez, essas informações auxiliam a empresa a ajustar sua postura tecnológica, seus investimentos em fontes internas e externas de tecnologia e o seu portfolio de tecnologias de produto e de processo. Isto orientaria a ampliação dos recursos necessários ao desenvolvimento e à introdução de produtos e processos novos ou melhorados tecnologicamente, viabilizando a exploração de novos segmentos de mercado ou dos já existentes. Diante disso, espera-se que as empresas com DIs mais altos devotem mais tempo e recursos à previsão tecnológica preliminar voltada para monitoramento do perfil de produtos e processos em comparação com aquelas que obtiveram, no mesmo período, DIs mais baixos. Ainda segundo Zahra (1996), em ambientes dinâmicos, essa previsão tecnológica preliminar é indispensável para antecipar descontinuidades tecnológicas. Por esta razão, empresas em ambientes dinâmicos devem dedicar mais tempo e recursos à previsão tecnológica preliminar voltada para monitoramento do perfil de produtos e processos do que empresas em ambientes mais estáveis.

Por fim, apresenta-se a perspectiva em relação aos investimentos realizados no sistema produtivo para torná-lo mais eficiente em termos de rapidez e custos operacionais. Nota-se que os autores que analisam empresas em países em desenvolvimento, tais como Bell et al. (1984), Dahlman et al, (1987) e Fleury (1988), e exatamente por esta razão, ao abordarem as estratégias tecnológicas adotadas, não têm se ocupado com aspectos voltados essencialmente para a criação de novos produtos e relacionados exclusivamente às atividades internas de pesquisa e desenvolvimento, desenvolvidas em departamentos estruturados nas empresas. As análises desses autores têm focalizado os esforços sistemáticos realizados por essas empresas para ampliar as suas capacidades de produção (ALVES FILHO, 1991).

Apesar de atribuir-se maior importância à capacidade de produção quando o mercado é pequeno, está estável ou em declínio, ela não se torna desprezível em ambientes dinâmicos (ALVES FILHO, 1991). Como exposto por Tidd et al. (2001), não basta às empresas que operam em mercados dinâmicos a capacidade de repor freqüentemente produtos novos ou melhorados, é preciso também produzi-los mais rápido do que os concorrentes e a preços mais baixos.

Frente a essa necessidade de lançar no mercado produtos a preços mais baixos e antes que os concorrentes, a expectativa é que o capital investido na produção (compra de equipamentos), em relação ao faturamento, nas empresas que possuem níveis de DI mais altos, supere aquele investido pelas empresas que possuem níveis de DI mais baixos. Esses investimentos, então, visariam, sobretudo, tornar o processo produtivo mais eficiente, tanto em termos de eficiência técnica quanto em termos de eficiência fator-preço. A primeira mede a economia no uso de recursos para produzir uma saída especificada, dado certo estado tecnológico. A segunda mede a habilidade em se conseguir a melhor combinação de diferentes recursos, tendo em vista seus preços relativos (ALVES F., 1991).

Essas são algumas das expectativas deduzidas da argumentação encontrada na literatura investigada para a realização da pesquisa aqui relatada. A seguir, é apresentado brevemente o método aplicado para o desenvolvimento da pesquisa de campo e no tópico seguinte são apresentados os resultados das análises estatísticas dos dados coletados nas empresas investigadas sobre os aspectos da ET aqui descritos.

\section{MÉTODO}

\section{Levantamento das empresas produtoras de equipamentos médico-hospitalares de São Carlos e Ribeirão Preto}

De acordo com a Classificação Nacional das Atividades Econômicas (CNAE), a indústria de equipamentos médico-hospitalares (Divisão 33, Grupo 33.1, Classe 33.10-3) é formada por empresas produtoras de aparelhos e instrumentos para usos médico-hospitalares, odontológicos e de laboratórios e aparelhos ortopédicos (CNAE, 1997:37).

Para a identificação das empresas produtoras de equipamentos médico-hospitalares localizadas em Ribeirão Preto, uma relação das empresas (produtoras e comerciantes) desse setor foi fornecida pela CODERP - Companhia de Desenvolvimento Econômico de Ribeirão Preto. Em seguida, entrou-se em contato com todas as empresas para verificar quais delas eram produtoras de equipamentos ou instrumentos médicos ou odontológicos e não apenas comerciantes destes produtos. Por meio deste procedimento encontrou-se um total de trinta e três empresas. Destas 33 empresas levantadas em Ribeirão Preto, 27 se dispuseram a colaborar com a pesquisa.

No caso das empresas industriais do setor médico-hospitalar localizadas em São Carlos, obteve-se, primeiramente, junto à prefeitura da cidade, o "Relatório de Empresas por Atividade Municipal". Neste documento figuram apenas os nomes das empresas localizadas em São Carlos (aproximadamente 600 empresas) e seus respectivos endereços, sem qualquer referência ao setor a que pertencem. Foram obtidos, então, os números dos telefones dessas empresas, via lista telefônica, e entrou-se em contato para verificar quais empresas eram produtoras de equipamentos ou instrumentos médicos, de laboratório ou odontológicos. Desta forma, encontrou-se um total de 15 empresas, e 12, dentre elas, se dispuseram a colaborar com a pesquisa.

Um questionário estruturado, com respostas circunscritas a uma escala de cinco pontos, foi o meio utilizado para conduzir a pesquisa junto às empresas, de maneira a aumentar a 
objetividade da coleta de dados e, conseqüentemente, facilitar o processo de análise de alguns aspectos das estratégias tecnológicas adotadas por essas empresas. Em outras palavras, as respostas dos entrevistados foram convertidas, durante a aplicação do questionário (aos entrevistados), em categorias expressas numericamente, o que permitiu que os dados fossem contados, tabulados e analisados estatisticamente.

\section{Classificação das empresas investigadas quanto ao desempenho inovador}

Como exposto na introdução deste artigo, os DIs das empresas investigadas podem ser medidos pelos seguintes indicadores: (1) quantidade de inovações em produto, resultantes dos esforços tecnológicos despendidos pelas empresas; e (2) parcela do faturamento proveniente da comercialização dos produtos que embutem essas inovações (INP - Índice de Novos Produtos). Este indicador referente à parcela do faturamento anual proveniente de produtos novos lançados no mercado nos últimos anos é calculado a partir da seguinte fórmula (SBRAGIA et al. 1998:392):

$\mathbf{I N P}=\frac{\begin{array}{c}\text { Faturamento anual gerado por produtos } \\ \text { com menos de cinco anos de vida }\end{array}}{\text { Faturamento anual da empresa }} \times \mathbf{1 0 0}$

Este índice de novos produtos será utilizado considerando-se os últimos três anos, ao invés de os últimos cinco anos. Para separar as 39 empresas investigadas em dois grupos, empresas com maiores e empresas com menores DIs, foi utilizada, primeiramente, a mediana da distribuição dos valores dos INPs apresentados por essas empresas, correspondente a $40 \%$ do faturamento anual. Seguiu-se aqui o procedimento usado por Sbragia et al. (1998) que, para separar um conjunto de 263 empresas que reportaram seus dados à ANPEI, referentes ao ano base 1996 e pertencentes a diversos segmentos industriais, em empresas menos e mais inovativas, utilizou a mediana da distribuição dos valores dos INPs.

Assim, no caso das empresas do setor de equipamentos médico-hospitalares analisadas, a princípio, o grupo denominado "empresas com menores DIs" ficaria composto por 20 empresas, com um INP médio equivalente a 18,9\% (desvio padrão igual a 11,5\%). O outro grupo, por sua vez, denominado "empresas com maiores DIs", ficaria composto por 19 empresas, com um INP médio equivalente a 78,1\% (desvio padrão igual a 15,6\%).

Concomitantemente, foi utilizado também o indicador "número de inovações em produtos lançados pelas empresas nos últimos três anos". Mais uma vez, para separar as empresas nos grupos com maiores e com menores DIs, recorreu-se à mediana da distribuição desse indicador, obtendo-se um valor correspondente a cinco produtos "novos".

Há que se ressaltar que, levando-se em conta as condições específicas de um país em desenvolvimento como o Brasil, há pouco mais de uma década livre de generalizada proteção comercial (SUZIGAN, 1992), são considerados produtos "novos" os que embutem inovações tanto de natureza significativa como de natureza incremental. Tendo como referência apenas este último critério, o grupo denominado "empresas com menores DIs" ficaria composto também por 20 empresas, com um número médio de 2,35 produtos "novos" (desvio padrão igual a 1,57) lançados no mercado nos últimos três anos. E o grupo denominado "empresas com maiores DIs" ficaria composto por 19 empresas, com um número médio de 12,8 produtos "novos" (desvio padrão igual a 6,79).

Aplicando, então, os dois critérios simultaneamente, obtiveram-se 14 e 13 empresas classificadas respectivamente nos grupos das empresas com menores e com maiores desempenhos. Isto é, 14 empresas lançaram no mercado, de 2001 a 2003, até quatro produtos "novos" e obtiveram uma parcela do seu faturamento proveniente da comercialização desses produtos igual ou inferior a $40 \%$. Da mesma forma, 13 empresas lançaram no mercado, de 2001 a 2003, cinco ou mais produtos novos e obtiveram uma parcela do seu faturamento proveniente da comercialização desses produtos superior a 40\%. As 12 empresas restantes não se encaixaram em nenhum dos dois grupos, aplicando-se, simultaneamente, os dois critérios de classificação. Por este motivo, não entraram na análise da relação entre ET e DI apresentada na seção seguinte.

\section{RESULTADOS}

Para a verificação da hipótese que norteia este trabalho, os dados sobre as ETs coletados junto às 27 empresas que puderam ser classificadas como sendo com maiores ou com menores DIs (27 dentre as 39 empresas pesquisadas) foram

Tabela 1: Gastos com atividades internas de P\&D\&E em relação ao faturamento da empresa.

\begin{tabular}{|l|c|c|}
\hline & MÉDIA & DESVIO PADRÃO \\
\hline Maior DI & $7,69 \%$ & $4,23 \%$ \\
\hline Menor DI & $1,27 \%$ & $1,65 \%$ \\
\hline
\end{tabular}

Independent Simples t-Test $(\mathrm{p}<0,05)$. 
contados, tabulados e analisados estatisticamente. Todas as variáveis paramétricas que compõem a análise das ETs, conforme definidas neste trabalho, foram analisadas (comparadas para os casos das empresas com maiores e menores desempenhos) através do método estatístico Independent Simples $t$-Test, e as variáveis não-paramétricas foram analisadas através do método estatístico Mann-Whitney, adotando-se para ambos a significância de $5 \%(\mathrm{p}<0,05)$. A seguir são apresentados e comentados alguns desses resultados.

Primeiramente, havia a expectativa de que as empresas com maiores DIs investissem mais em atividades internas de P\&D\&E do que aquelas com menores DIs. Esta expectativa foi corroborada, como mostram os dados da Tabela 1.

Enquanto as empresas com maiores DIs investem, em média, 7,69\% de seus faturamentos em atividades internas de P\&D\&E, as com menores desempenhos investem apenas $1,27 \%$. Estes resultados, portanto, vão ao encontro do exposto em outros trabalhos empíricos sobre a relação entre ET e desempenho das empresas, que mostram a influência positiva dos investimentos em atividades internas de P\&D e também de engenharia no desempenho das empresas (DOWLING; MCGEE, 1994; DOWLING; RUEFLI, 1992; PEGELS; THIRUMURTHY, 1996; ZAHRA, 1996).

Essa influência se dá através da incorporação de inovações resultantes desses esforços de P\&D\&E nos produtos comercializados pela empresa, ajudando-as a capturar e manter parcelas de mercado e a aumentar sua lucratividade.

Constatou-se também, com base na avaliação do dinamismo tecnológico do setor no qual a empresa atua feita pelos próprios entrevistados, que as empresas classificadas como sendo aquelas com maiores DIs encontram-se em ambientes ainda mais dinâmicos tecnologicamente do que aquelas classificadas como com menores DIs, o que levaria as primeiras empresas a investirem mais em atividades de P\&D\&E devido à necessidade também maior, imposta pelo próprio ambiente, de reporem freqüentemente produtos novos ou melhorados tecnologicamente.

Frente também a essa necessidade de estarem constantemente inovando, e frente às dificuldades das empresas que atuam em economias em desenvolvimento para terem acesso às "tecnologias superiores", a expectativa era que os investimentos em P\&D\&E fossem voltados sobretudo para inovações incrementais em produtos. Os dados das Tabelas 2 e 3 mostram que essa expectativa também foi corroborada.

Constata-se na Tabela 2 que tanto as empresas com maiores quanto as com menores DIs realizam suas atividades internas de $\mathrm{P} \& \mathrm{D} \& \mathrm{E}$ voltadas principalmente para produto.

No caso específico do grupo de empresas com maiores DIs, a proporção das que investem em atividades de $\mathrm{P} \& \mathrm{D} \& \mathrm{E}$ voltadas para produto e para processo é superior à das que investem apenas em atividades de P\&D\&E voltadas para produto. De acordo com Quadros et al. (1999), que obtiveram o mesmo resultado ao analisar o comportamento inovativo das empresas paulistas através do questionário PAEP-1996, essa dinâmica confirma a hipótese neoschumpeteriana acerca da natureza cumulativa do aprendizado tecnológico.

Como resultados desses investimentos, tem-se, na Tabela 3 , as médias dos números de inovações incrementais e significativas realizadas pelas empresas com maiores e pelas empresas com menores DIs.

Primeiro, cabe constatar que o número de inovações significativas realizadas pelas empresas com maiores DIs supera aquele realizado pelas empresas com menores DIs.

Tabela 2: Percentagem das despesas em P\&D\&E destinadas a inovações em produto e processo.

\begin{tabular}{|l|c|c|c|c|}
\hline & \multicolumn{2}{|c|}{ PRODUTO } & \multicolumn{2}{c|}{ PROCESSO } \\
\hline & MÉDIA & DESVIO PADRÃO & MÉDIA & DESVIO PADRÃOO \\
\hline Maior DI & $77,7 \%$ & $22 \%$ & $22,3 \%$ & $22 \%$ \\
\hline Menor DI & $53,8 \%$ & $51,9 \%$ & $0 \%$ & $0 \%$ \\
\hline
\end{tabular}

Independent Simples t-Test (Produto $(p<0,05)$; Processo $(p<0,05)$ ).

Tabela 3: Inovações em produto.

\begin{tabular}{|l|c|c|c|c|}
\hline & \multicolumn{2}{|c|}{ № DE INOVAÇÕES INGREMENTAIS } & \multicolumn{2}{c|}{ No DE INOVAÇÕES SIGNIFIGATIVAS } \\
\hline & MÉDIA & DESVIO PADRÃO & MÉDIA & DESVIO PADRÃO \\
\hline Maior DI & 8,38 & 5,08 & 4,15 & 3,87 \\
\hline Menor DI & 1,5 & 1,02 & 0,21 & 0,43 \\
\hline
\end{tabular}

Independent Simples t-Test $(p<0,05)$. 
Segundo, a maioria das inovações em produtos realizados pelas empresas dos dois grupos é de caráter incremental.

Portanto, mesmo ao analisar apenas aquelas empresas com maiores DIs, os resultados revelam um comportamento de inovação predominantemente defensivo, que tem como característica dominante o desenvolvimento de inovações de produto de natureza incremental. Desta forma, também aquelas empresas com maiores DIs esforçam-se principalmente em suprir as necessidades do mercado (demand pull), e não em antecipar essas necessidades e explorar oportunidades tecnológicas (technology push).

Outros resultados que vão ao encontro desse comportamento de inovação predominantemente defensivo são aqueles referentes à estruturação dos departamentos de $\mathrm{P} \& \mathrm{D} \& \mathrm{E}$ nas empresas investigadas (Tabela 4).

Tanto no caso das empresas com maiores quanto no caso das com menores DIs, os departamentos de P\&D\&E são informalmente organizados na maioria das empresas, e essas atividades esporádicas de P\&D\&E são geralmente realizadas para atender necessidades do mercado.

Sobre as fontes externas de tecnologia, como a maioria das empresas investigadas é micro ou de pequeno porte e as atividades de P\&D\&E necessitam de investimentos significativos, às vezes até proibitivos para essas empresas, a expectativa era de que a maioria das empresas utilizasse preferencialmente fontes externas de tecnologia. E, conseqüentemente, que essas empresas investissem mais em aquisição de tecnologias a partir de fontes externas do que em atividades internas de $P \& D \& E$.

Os dados da Tabela 5 revelam os investimentos feitos pelas empresas com maior e com menor DI na aquisição de tecnologias a partir de fontes externas.

Se forem comparados esses dados com aqueles da Tabela
1 , verifica-se que tanto as empresas com maiores quanto as com menores DIs vêm investindo mais em P\&D\&E interno do que na aquisição de tecnologias a partir de fontes externas. Isto é, a expectativa de que os investimentos em fontes externas de tecnologia fossem superiores aos investimentos realizados em atividades internas de $P \& D \& E$ não foi confirmada.

Estes maiores investimentos em atividades internas de P\&D\&E do que em fontes externas de tecnologia indicam que a estratégia de reprodução de produtos e processos já existentes no mercado não garante a sobrevivência da empresa no longo prazo sem que simultaneamente sejam realizados investimentos em atividades internas de P\&D\&E. Mesmo que a empresa adquira tecnologias desenvolvidas por terceiros, com freqüência haverá a necessidade de adaptar essa tecnologia às condições de conhecimento da empresa, o que requer que atividades de P\&D\&E sejam levadas a cabo. Assim, as atividades de P\&D\&E são realizadas tanto para o desenvolvimento de inovações significativas ou incrementais em produtos e processos, totalmente e unicamente dentro da empresa, quanto para dar suporte à aquisição de tecnologias desenvolvidas por terceiros que vão ser incorporadas aos produtos e processos da empresa.

Além disso, o fato de que a maioria das empresas realiza atividades de P\&D\&E de forma esporádica, e deste modo não mantêm departamentos de P\&D\&E estruturados, talvez seja uma das causas de recorrerem mais ao P\&D\&E interno do que às fontes externas de tecnologia, pois os gastos com essas atividades ocasionais não se tornam tão proibitivos para essas pequenas empresas.

Por outro lado, a hipótese de que as empresas com maiores DIs investissem mais na aquisição de tecnologias a

Tabela 4: Formas de realização das atividades internas de P\&D\&E.

\begin{tabular}{|l|c|c|}
\hline & MAIOR DI & MENOR DI \\
\hline$\%$ de empresas com P\&D\&E estruturado & $38,46 \%$ & $14,28 \%$ \\
\hline$\%$ de empresas com P\&D\&E semi-estruturado latividades esporádicas de P\&D\&E] & $61,54 \%$ & $42,86 \%$ \\
\hline$\%$ de empresas que não realizam atividades de P\&D\&E & $0 \%$ & $42,86 \%$ \\
\hline
\end{tabular}

Mann-Whitney $(p<0,05)$.

Tabela 5: Gastos com aquisição de tecnologias de produto e processo a partir de fontes externas em relação ao faturamento da empresa.

\begin{tabular}{|l|c|c|}
\hline & MÉDIA & DESVIO PADRÃO \\
\hline Maior DI & $2,269 \%$ & $2,93 \%$ \\
\hline Menor DI & $0,14 \%$ & $0,29 \%$ \\
\hline
\end{tabular}

Independent Simples t-Test $(p<0,05)$. 
partir de fontes externas, em comparação com aquelas com menores DIs, foi corroborada, como mostram os dados da Tabela 5 .

Aliás, a soma dos investimentos em atividades internas de P\&D\&E e em fontes externas de tecnologia é superior no caso das empresas com maiores DIs em comparação com as empresas com menores desempenhos. Com efeito, parece lógico que as empresas que lançaram maior número de "novos" produtos no mercado nos últimos três anos tiveram que investir mais no desenvolvimento desses produtos, sejam estes desenvolvidos através das atividades internas de P\&D\&E ou desenvolvidos fora da empresa e por ela adquiridos.

Cabe ressaltar que essas empresas, quando investem em fontes externas de tecnologia, visam principalmente ampliar as competências e os recursos tecnológicos necessários ao desenvolvimento de produtos e processos novos ou melhorados.

Com este propósito, no caso das empresas com menores desempenhos, os meios externos mais utilizados para o desenvolvimento de "novos" produtos são as alianças de cooperação com fornecedores. Por outro lado, os meios menos utilizados são a compra e o licenciamento de novas tecnologias desenvolvidas por outras empresas e as alianças de cooperação com empresas estrangeiras. Já para desenvolvimento de novos processos ou melhorias nos existentes, as empresas com menores desempenhos praticamente não utilizam qualquer fonte externa de tecnologia.

Em relação às empresas com maiores DIs, as principais fontes externas por elas utilizadas são as contratações de outras empresas, universidades ou centros de pesquisa para desenvolver tecnologias de produto. Em seguida estão as alianças de cooperação com consumidores, com universidades e centros de pesquisa e, por fim, com fornecedores, para o desenvolvimento de novos produtos e melhorias tecnológicas em produtos já comercializados pela empresa.

Por outro lado, os recursos menos utilizados, do mesmo modo como no caso das com menores DIs, são a compra e o licenciamento de tecnologias desenvolvidas por terceiros e as alianças de cooperação com empresas estrangeiras.

Por sua vez, para desenvolver processos tecnologicamente novos ou melhorados, as fontes externas mais utilizadas são as alianças com fornecedores, seguidas pelas contratações de outras empresas, universidades ou centros de pesquisa.

Em relação ao portfolio de tecnologias, esperava-se que as empresas com maiores DIs detivessem a maioria das tecnologias básicas de produto e de processo necessárias a qualquer empresa para competir naquela indústria específica e um número considerável de tecnologias chaves.

Por outro lado, esperava-se que as empresas com menores DIs não detivessem a maioria das tecnologias básicas de produto e de processo e um conjunto expressivo de tecnologias chaves. Tais expectativas foram confirmadas em parte, como mostram os dados das Tabelas 6 e 7.

Essas tabelas mostram o número de empresas, dentro dos grupos daquelas com maiores e com menores DIs, que

Tabela 6: Portfolio de tecnologias de produto.

\begin{tabular}{|l|c|c|c|c|c|c|}
\hline & \multicolumn{2}{|c|}{ TECNOLOGIAS GHAVES PRODUTO } & \multicolumn{3}{c|}{ TECNOLOGIAS BÁSICAS PRODUTO } \\
\hline & BAIXA & MÉDIA & ALTA & BAIXA & MÉDIA & ALTA \\
\hline $\begin{array}{l}\text { № de empresas com } \\
\text { maiores Dls }\end{array}$ & 1 & 5 & 7 & 0 & 2 & 11 \\
\hline $\begin{array}{l}\text { № de empresas com } \\
\text { menores Dls }\end{array}$ & 10 & 2 & 2 & 1 & 8 & 5 \\
\hline
\end{tabular}

Mann-Whitney $(p<0,05)$

Tabela 7: Portfolio de tecnologias de processo.

\begin{tabular}{|l|c|c|c|c|c|c|}
\hline & \multicolumn{2}{|c|}{ TECNOLOGIAS CHAVES PROCESSO } & \multicolumn{2}{c|}{ TECNOLOGIAS BÁSICAS PROGESSO } \\
\hline & BAIXA & MÉDIA & ALTA & BAIXA & MÉDIA & ALTA \\
\hline $\begin{array}{l}\text { № de empresas com } \\
\text { Maiores Dls }\end{array}$ & 3 & 6 & 4 & 1 & 2 & 10 \\
\hline $\begin{array}{l}\text { № de empresas com } \\
\text { Menores Dls }\end{array}$ & 10 & 3 & 1 & 2 & 6 & 6 \\
\hline
\end{tabular}

Mann-Whitney (Tecnologias chaves $(p<0,05)$; Tecnologias básicas $(p<0,05)$ ). 
possuem uma quantidade baixa, média ou alta de tecnologias chaves e básicas de produto e processo geradas pela empresa ou que ela tenha adquirido a partir de fontes externas.

Pode-se observar que a maioria das empresas com maiores DIs possui uma quantidade alta de tecnologias básicas de produto e uma quantidade média/alta de tecnologias chaves. Enquanto que a maioria das empresas com menores DIs possui uma quantidade média/alta de tecnologias básicas de produto e uma quantidade baixa de tecnologias chaves. seu dinamismo tecnológico, como o químico e o eletroeletrônico. Também são considerados nesta análise os contatos com fornecedores, consumidores e outras empresas do setor para a troca de informações sobre as novidades tecnológicas no setor de equipamentos médico-hospitalares e a assinatura de revistas científicas que trazem novidades no setor e em setores correlatos.

Fazendo, então, uma reflexão sobre esses quesitos, a própria empresa classificou a freqüência e magnitude com que ela estava a par das mudanças tecnológicas na indústria em que atua e nas indústrias correlacionadas como: muito baixa, baixa, média, alta, muito alta ou não faz monitoramento do desenvolvimento tecnológico do seu setor e de setores correlatos. A Tabela 8 mostra os resultados obtidos.

Como mostram os dados da Tabela 8 , embora as empresas de ambos os grupos se dediquem à previsão tecnológica preliminar voltada para monitoramento do perfil de produtos e processos, as

Em relação às tecnologias de processo, a diferença entre os dois grupos está nas tecnologias chaves. Enquanto as empresas com maiores DIs, em sua grande maioria, detêm uma quantidade média/alta de tecnologias chaves de processo, as com menores desempenhos detêm uma quantidade média/baixa.

Já a maioria das tecnologias básicas de processo necessárias a qualquer empresa para competir naquela indústria específica é encontrada na maior parte das empresas com maiores e com menores DIs.

Outro aspecto analisado em relação às ETs adotadas pelas empresas com maiores e com menores desempenhos é a previsão tecnológica preliminar voltada para monitoramento do perfil de produtos e processos, referente ao monitoramento que a empresa faz do desenvolvimento tecnológico em seu setor de atuação e em setores correlatos. Este monitoramento é analisado aqui pela participação das empresas em congressos e feiras relacionadas ao setor de equipamentos médico-hospitalares e àqueles setores que dão suporte ao empresas com maiores DIs o fazem com uma freqüência e magnitude maior.

Como exposto anteriormente, com base na avaliação do dinamismo tecnológico do setor no qual a empresa atua feita pelos próprios entrevistados, as empresas com maior desempenho tecnológico encontram-se em ambientes mais dinâmicos tecnologicamente do que as com menores DIs e, segundo Zahra (1996), a previsão tecnológica preliminar em tais ambientes torna-se indispensável à sobrevivência dessas empresas. Isto, então, contribuiria para entender esse intenso monitoramento que a maioria das empresas investigadas com maiores DIs faz do desenvolvimento tecnológico em seu setor de atuação e em setores correlatos.

A última expectativa em relação às ETs adotadas pelas empresas com maiores e com menores DIs é sobre o capital investido na produção. Estão incluídos nestes investimentos essencialmente aqueles gastos com a compra de equipamentos para o sistema produtivo da empresa, visando torná-lo mais eficiente, em termos de rapidez e custos operacionais.

Tabela 8: A freqüência e a magnitude com que a empresa está a par das mudanças tecnológicas na indústria em que atua e nas indústrias correlacionadas.

\begin{tabular}{|l|c|c|c|c|c|}
\hline & BAIXA & MÉDIA & ALTA & MUITO ALTA & NÃO FAZ \\
\hline $\begin{array}{l}\text { № de empresas com } \\
\text { Maiores Dls }\end{array}$ & 0 & 1 & 3 & 9 & 0 \\
\hline $\begin{array}{l}\text { № de empresas com } \\
\text { Menores Dls }\end{array}$ & 0 & 3 & 8 & 3 & 0 \\
\hline
\end{tabular}

Mann-Whitney $(p<0,05)$. 
Deste modo, frente à necessidade das empresas que operam em ambientes tecnologicamente dinâmicos não só de repor freqüentemente produtos "novos", mas também de lançá-los no mercado a preços mais baixos e antes que os concorrentes, a expectativa era que o capital investido na produção, em relação ao faturamento, nas empresas que possuem maiores DIs, superasse aquele investido pelas empresas que possuem menores DIs.

Entretanto, essa hipótese não foi corroborada, ou seja, não há estatisticamente diferença significativa entre os investimentos realizados pelas empresas com maiores e com menores DIs. Cabe, por fim, observar que as empresas em geral investiram, nestes três últimos anos, em média, 9,77\% $($ desvio padrão $=13,5)$ dos seus faturamentos com compra de novos equipamentos para o sistema produtivo.

Estes, portanto, são os resultados em relação à comparação entre as estratégias tecnológicas adotadas pelas empresas investigadas com maiores e com menores DIs. Na seção seguinte, faz-se uma síntese das diferenças (e algumas semelhanças) encontradas entre as ETs adotadas por esses dois grupos de empresas.

\section{CONCLUSÕES}

No presente artigo, foram avaliados alguns aspectos das ETs adotadas por pequenas e médias empresas do setor de equipamentos médico-hospitalares localizadas nas cidades de São Carlos e Ribeirão Preto. Na análise empreendida, foram encontradas as seguintes diferenças nas ETs entre o grupo daquelas que obtiveram níveis mais altos e o grupo das que obtiveram níveis mais baixos de DI:

- As empresas com maiores DIs investem mais em atividades internas de P\&D\&E do que aquelas com menores DIs;

- As empresas com maiores DIs investem mais na aquisição de tecnologias a partir de fontes externas em comparação com aquelas com menores DIs;

- Os recursos externos mais utilizados pelas empresas com menores desempenhos para o desenvolvimento de "novos" produtos são as alianças de cooperação com fornecedores. Ao passo que as com maiores desempenhos fazem uso principalmente das contratações de outras empresas, universidades ou centros de pesquisa para desenvolver tecnologia de produto;

- Para desenvolver processos tecnologicamente novos ou melhorados, as fontes externas mais utilizadas pelas empresas com maiores DIs são as alianças com fornecedores, seguidas pelas contratações de outras empresas, universidades ou centros de pesquisa. Já as empresas com menores DIs praticamente não utilizam qualquer fonte externa de tecnologia para esse fim;

- Enquanto as empresas com maiores DIs, em sua grande maioria, detêm uma quantidade média/alta de tecnologias chaves de processo, as com menores desempenhos detêm uma quantidade média/baixa;

- As empresas com maiores e com menores DIs se dedicam à previsão tecnológica preliminar voltada para monitoramento do perfil de produtos e processos, porém, as empresas com maiores DIs o fazem com uma freqüência e magnitude ainda maior; e

- O número de inovações significativas realizadas pelas empresas com maiores DIs supera aquele realizado pelas empresas com menores DIs.

Constatou-se também que:

- Tanto as empresas com maiores quanto as com menores DIs realizam suas atividades internas de P\&D\&E voltadas principalmente para produto;

- Tanto no caso das empresas com maiores quanto das com menores DIs, os departamentos de P\&D\&E são informalmente organizados (P\&D\&E semi-estruturado) na maioria das empresas;

- Tanto as empresas com maiores quanto as com menores DIs vêm investindo mais em $P \& D \& E$ interno do que na aquisição de tecnologias a partir de fontes externas; e

- A maioria das inovações em produtos realizada pelas empresas dos dois grupos é de caráter incremental.

Com base nestes resultados, tem-se indícios de que os padrões de ET seguidos pelas empresas com maiores DIs, que se diferenciaram dos padrões seguidos pelas empresas com menores DIs, tais como maiores investimentos em atividades internas de P\&D\&E e em fontes externas de tecnologia, maior dedicação à previsão tecnológica preliminar voltada para monitoramento do perfil de produtos e o desenvolvimento de um amplo portfolio de tecnologias de produto e de processo, lhes permitem alcançar maiores DIs.

Como ressaltado na introdução deste artigo, a análise aqui empreendida procura contribuir para o estudo da relação conjunta entre estratégia, tecnologia e desempenho, uma vez que há uma escassez de estudos empíricos sobre a dinâmica da relação entre ET e desempenho de empresas. Visa também propiciar melhor compreensão do comportamento inovativo das empresas produtoras de equipamentos médico-hospitalares localizadas em São Carlos e em Ribeirão Preto.

Conhecer esse comportamento é fundamental para a elaboração de políticas e a implementação de instrumentos públicos e privados voltados à promoção das atividades dessas empresas que se mostram muito importantes para o desenvolvimento econômico e tecnológico das cidades onde se localizam - embora esse conhecimento seja apenas uma parte do necessário para promover tais políticas. Os governos municipais, via essas políticas, podem, por exemplo, 
estimular os processos de transferência de conhecimentos entre os agentes institucionais promotores de pesquisa e desenvolvimento, como as universidades locais, e as empresas locais do setor de equipamentos médico-hospitalares. Também em uma perspectiva de ação estratégica mais ampla, o governo municipal e as empresas locais do setor de equipamentos médico-hospitalares podem empenhar-se na proposição de projetos de desenvolvi- mento da estrutura produtiva do setor.

Além disso, esse conhecimento sobre as empresas estudadas do setor de equipamentos médico-hospitalares de São Carlos e Ribeirão Preto, embora todas se localizem numa mesma região geográfica, contribui para o entendimento das atividades empresariais de inovação tecnológica desse setor no país, uma vez que há poucos trabalhos acadêmicos a respeito dessa questão.

\section{Artigo recebido em 08/11/2005 Aprovado para publicação em 25/05/2007}

\section{- Referências}

ALBUQUERQUE, E. M.; CASSIOLATO, J. E. As especificações do sistema de inovação do setor de saúde: uma resenha da literatura como introdução a uma discussão sobre o caso brasileiro. Estudos FeSBE I. São Paulo - Universidade Estadual de São Paulo, 2000.

ALVES FILHO, A. G. Estratégia Tecnológica, Desempenho e Mudança: estudo de caso em empresas da indústria de calçados. São Paulo, 1991. Tese (Doutorado em Engenharia de Produção) - Escola Politécnica, Universidade de São Paulo.

BELL, R. M. "Learning" and the accumulation of industrial technological capacity in developing countries. In: Fransman, M.; King, K. Technological capability in the third world. New York: St Martin's Press, 1984.

BURGELMAN, R. A.; ROSENBLOOM, R. $S$. Technology strategy: an evolutionary process perspective. In: Burgelman, R. A. \& Rosenbloom, R. S (Eds), Research on Technological Innovation, Management and Policy. Greenwich: JAI Press, 1989, p. 1-23.

CNAE - Classificação Nacional das Atividades Econômicas, 1997. Disponível em: www.ibge.org.br

DAHLMAN, C. J.; ROSS-LARSON, B.; WESTPHAL, L. E. Managing technological development: lessons from the newly industrializing countries. World Development, v. 15 , n. 6, p. 759-775, 1987.

DEEDS, D. L.; DECAROLIS, D.; COOMBS, J. E. The impact of firm-specific capabilities on the amount of capital raised in an initial public offering: evidence from the biotechnology industry. Journal of Business Venturing, v. 12, p. 31-46, 1997.

DOWLING, M. J.; MCGEE, J. Using R\&D cooperative arrangements to leverage managerial experience: a study of technology-intensive new ventures. Journal of Business Venturing, v. 9, n. 1, p. 33-48. 1994

DOWLING, M. J.; RUEFLI, T. W. Technological innovation as a gateway to entry: the case of the telecommunication industry. Research Policy. n. 21, p. 63-77, 1992.

FERNANDES, A. C.; CÔRTES, M. R. Caracterização do perfil da pequena empresa de base tecnológica no estado de São Paulo: uma análise preliminar. In SCIENCE AND TECHNOLOGY RESEARCH SEMINAR, 1998, Campinas. Anais... Campinas: Unicamp - Instituto de Geociências, 1998.

FERNANDES, A. C.; et al. Potencialidades e limites para o desenvolvimento de empresa de base tecnológica no Brasil: contribuições para uma política setorial. São Carlos, 1999 (mimeo). Projeto FAPESP n.o 98/14127/ 0 - Departamento de Engenharia de Produção da UFSCar

FLEURY, A. C. C. Análise a nível de empresa dos impactos da microeletrônica sobre a organização da produção e do trabalho. São Paulo, 1988. Tese (professor titular) - Escola Politécnica, Universidade de São Paulo.
FURTADO, J. A indústria de equipamentos médico-hospitalares: elementos para uma caracterização da sua dimensão internacional. Campinas, 1999 (mimeo)

FURTADO, A. T.; SOUZA, J. H. Relatório final setor de equipamentos médicos - evolução do setor de insumos e equipamentos médicohospitalares, laboratoriais e odontológico brasileiro: a década de 90 . Campinas, junho de 2000 (mimeo) - Relatório Final - Departamento de Política Científica e Tecnológica - Instituto de Geociências - Unicamp.

GADELHA, C. A. G. Estudos de competitividade por cadeias integradas no Brasil: impactos das zonas de livre comércio Cadeia: complexo da saúde. Campinas, dezembro de 2002. Nota técnica finalDepartamento de Política Científica e Tecnológica - Instituto de Geociências Unicamp

GUIADE FORNECEDORES HOSPITALARES, 2001. 1a Revista Brasileira de Produtos, Serviços e Tecnologias de uso em Estabelecimentos de Saúde. Ano 7, n. 67, maio, 2001

HARRIS, R.C.; et al. The virtual R\&D laboratory. Research-Technology Management, p. 32-36, March/April, 1996.

HASENCLEVER, L.; CASSIOLATO, J. E. Capacitação tecnológica empresarial brasileira e transferência de tecnologia. In: SIMPÓSIO DE GESTÃO DA INOVAÇÃO TECNOLOGICA, XX, 1998, São Paulo. Anais... (CD Rom). São Paulo, 1998, p. 309-321.
KLEINKNECHT. A. H.; POOT, P.T.; REIJNEN, J. O. N. Formal and Informal R\&D and Firm Size: survey results from the Netherlands. In: ACS, Z. J.; AUDRETSCH, D. B. (eds.). Innovation and Technology Change. New York: Haverster/ Wheatsheaf, 1991.

LANCTOT, A. \& SWAN, K. S. Technology Acquisition Strategy in an Internationally Competitive Environment. Journal of International Management, v. 6, p. 187215, 2000.

MILLER, A. A taxonomy of technological settings, with related strategies and performance levels. Strategic Management Journal. v. 9, p. 239-254, 1988.

NEPP - NÚCLEO DE ESTUDOS DE POLÍTICAS PÚBLICAS. 0 setor de saúde e o complexo da saúde no Brasil. Caderno n.46, 2000. DPP/FINEP. I Relatório Setorial DPP/FINEP - Equipamentos médicos, hospitalares e odontológicos, 2004 (mimeo).

OLIVEIRA, J. P. Os aspectos tecnológicos necessários à formação de um cluster de empresas fabricantes de equipamentos médicos e odontológicos. Relatório de Iniciação Científica do PIBIC, 2002 (mimeo).

PAVITT, K.; BELL, M Technological accumulation and industrial growth: contrasts between developed and developing countries. Industrial and Corporate Change, v. 2, n. 2, p. 157-210, 1993. 


\section{Referências}

PEGELS, C.; THIRUMURTHY, M. V. The impact of technology strategy on firm performance. IEEE Transactions on Engineering Management, v. 43, n. 3, p. 346-349, 1996

QUADROS, R.; et al. Technological innovation in Brazilian industry: An assessment based on the São Paulo innovation survey. In: INTERNATIONAL CONFERENCE ON TECHNOLOGY POLICY AND INNOVATION, III, 1999, Austin. Annals... Austin, 1999.

SBRAGIA, R.; et al. Os indicadores de P\&D nas empresas mais e menos inovadoras. In: SIMPÓSIO DE GESTÃO DA INOVAÇÃO
TECNOLÓGICA, XX, São Paulo. Anais... (CD ROM). São Paulo, 1998, p. 388-400

SCHERER, F; HUH, K. R\&D reactions to high-technology import competition. Review of Economics and Statistics. n. 74 p. 202-212, 1992.

SOBEET - SOCIEDADE BRASILEIRA DE ESTUDOSDE EMPRESAS TRANSACIONAIS E DA GLOBALIZAÇÃO ECONÔMICA. Comportamento Tecnológico das Empresas Transacionais em Operação no Brasil. Conjuntura Econômica, mar. 2000.

SPICTAL, F. C.; BICKFORD, D. J. Successfu competitive and technology strategies in dynamic and stable product technology environments. Journal of Engineering and Technology Management. n. 9, p. 29-60, 1992.

SUZIGAN, W. A indústria brasileira após uma década de estagnação: questões para política industrial. Economia $e$ Sociedade, Campinas: IE-Unicamp, n. 1, p. 89-109, 1992.

TELLES, L. O. Cluster e a Indústria ligada à área da saúde em Ribeirão Preto. Ribeirão Preto, 2002. Dissertação de Mestrado Faculdade de Economia, Administração e Contabilidade da Universidade de São Paulo.
TIDD, J.; BESSANT, J.; PAVITT, K. Managing innovation: integrating technological, market and organizational change. 2 ed John Wiley \& Sons, LTD, 2001.

WILBON, A. D. An empirical investigation of technology strategy in computer software initial public offering firms. Journal of. Eng. Technology Management, v. 16, p. 147-169, 1999.

ZAHRA, S. A. Technology strategy and financial performance: examining the moderating role of the firm's competitive environment. Journal of Business Venturing, v. 11, p. 189-219, 1996.

\section{- Sobre os autores}

\section{Denise Luciana Rieg}

Departamento de Engenharia de Produção

Centro Universitário Fundação Santo André

End.: Av. Príncipe de Gales, 821 - CEP 09060-650 - Santo André - SP

E-mail:riegsc@fsa.br

\section{Alceu Gomes Alves Filho}

Departamento de Engenharia de Produção

Universidade Federal de São Carlos

Caixa Postal 676 - 13565-905 - São Carlos - SP

E-mail: alceu@power.ufscar.br 